Portland State University

PDXScholar

$5-24-2019$

\title{
Suicidal Ideation and Mindfulness Practice in LGBTQ Youth
}

McKenna R. Martin

Portland State University

Follow this and additional works at: https://pdxscholar.library.pdx.edu/honorstheses Let us know how access to this document benefits you.

\section{Recommended Citation}

Martin, McKenna R., "Suicidal Ideation and Mindfulness Practice in LGBTQ Youth" (2019). University Honors Theses. Paper 743.

https://doi.org/10.15760/honors.760

This Thesis is brought to you for free and open access. It has been accepted for inclusion in University Honors Theses by an authorized administrator of PDXScholar. Please contact us if we can make this document more accessible: pdxscholar@pdx.edu. 
Suicidal Ideation and Mindfulness Practice in LGBTQ Youth

by

\title{
McKenna Martin
}

An undergraduate honors thesis submitted in partial fulfillment of the requirements for the degree of

\author{
Bachelor of Science \\ in \\ University Honors \\ and \\ Child, Youth, and Family Studies
}

Thesis Adviser

Jana Meinhold

Portland State University 


\section{Introduction}

Mindfulness as therapeutic practice has been growing in usefulness the last decade, particularly when looking at the impact of mindfulness on depression (Deyo, Wilson, Ong, and Koopman, 2009). A small number of studies have extended beyond depression and focused on the impact of mindfulness on suicidal ideation, one of which were conducted with youth. There is need for further consideration and research surrounding mindfulness practice and LGBTQ youth (lesbian, gay, bisexual, transgender, queer - identified as ages 15-24) who experience suicidal ideation. There is also a need for suicide prevention programming to expand practitioners' strategies. Given the prevalence and devastating impact of suicide among LGBTQ youth, developing more effective ways to support youth, prevent attempts, and deaths is essential. There is relatively little research surrounding this particular population, and no research has been performed looking at using mindfulness practice as a tool for reducing suicidal ideation and related factors within this population. This paper is going to explain the significance of and draw together the areas of mindfulness practice and suicidal ideation, specifically among LGBTQ youth.

\section{Youth Suicide and LGBTQ Youth}

Youth suicide is a enormous, devastating, and pervasive issue in our country. According to the Centers for Disease Control (2016), suicide is the second leading cause of death for North American youth. In 2017 the suicide rate for youth aged 15-24 was a crude rate of 14.46 according to the American Foundation for Suicide Prevention. In 2016, ninety-eight Oregon youth between the ages of 10 and 24 died by suicide. Oregon's youth suicide rate is twice the 
national rate with LGBTQ youth at particularly high risk (Youth Suicide and Prevention Plan, 2017). This risk remains after controlling for other risk factors of suicide including alcohol abuse, depression, family history of suicide attempts, and prior victimization (Cash \& Bridge, 2009).

LGBTQ youth have in general more risk factors, risk factors that are more severe, and less protective factors than heterosexual youth according to the Suicide Prevention Resource Center (2008). These youth often lack protective factors including family support and a safe school environment. Forty-five percent of youth who attempted suicide in 2010 were associated with family discord, the most common factor associated with suicide attempts that year (Oregon Health Authority, 2012). LGBTQ individuals also face stigma and discrimination, which are two important factors that are directly tied to risk factors for suicide. Suicide attempts are significantly higher among LGBTQ youth than their heterosexual peers (Suicide Prevention Resource Center, 2008). A 2015 study of youth in the United States found that $38.2 \%$ of LGBTQ youth had made a plan for suicide within the past 12 months compared to $11.9 \%$ of non-LGBTQ youth (Centers for Disease Control and Prevention, 2016). Additionally, a study by Safren and Heimberg (1999) found that 58\% of LBG youth who had attempted suicide stated that they had truly hoped to die, in contrast to $33 \%$ of heterosexuals who had hoped to die on their reported suicide attempts.

Many risk factors have been identified as contributing to suicidal behavior for both youth and adults within the general population. Some of these risk factors are: stigma, depression, anxiety, exposure to suicide of a family member or friend, suicidal ideation, ruminative thinking, substance abuse, access to firearms and lethal means, impulsive aggression, stigma associated 
with seeking help, isolation and exclusion, and institutional discrimination (American Association of Suicidology, 2016; Little, Roche, Chow, Schenck, \& Byam, 2016; Luoma \& Villatte, 2011; Suicide Prevention Resource Center, 2008). Specifically for LGBTQ youth, widespread discimination against the LGBTQ community, heterosexist attitudes, and gender bias can place stress on the individual and also lead to other risk factors such as isolation, family rejection, and lack of access to care providers. This stress can turn into depression and substance abuse, which puts these youth at a higher risk for suicide. Gender non-conformity was also associated with greater self-harm (Liu \& Mustanski, 2012). Liu and Mustanski (2012) found that general as well as LGBTQ specific risk factors contributed uniquely to the likelihood of suicidal ideation and self-harm in LGBTQ youth. According to the Suicide Prevention Resource Center (2008), studies have shown that internalized homophobia and conflict about sexual orientation lead to higher suicide risk among LGBTQ youth. On the other side, protective factors that contribute to an individual being less likely to commit suicide include restricted access to lethal means, community and family support, access to care providers, and coping skills. Mindfulness practice has been identified as a coping skill for suicidal ideation because it reduces ruminative thinking, which fuels suicidal ideation.

\section{Suicidal Ideation}

Suicidal ideation is one factor that the majority of people who attempt or die by suicide have experienced, making suicide ideation important to address and study. Suicidal ideation is defined as the thinking about or planning of suicide. Suicidal ideation can range in its severity from fleeting, passing thoughts, to a detailed plan for attempting suicide (Suicide Prevention 
Resource Center, 2008). Twenty-three percent of the 84,418 youth and adults ages 13 and up that filled out the PHQ-9 (questionnaire for screening, diagnosing, and measuring the severity of depression) indicated on their first assessment that they were experiencing suicidal ideation. This suicidal ideation was linked with a greater risk for future suicide attempts and completion (Simon, Rutter, Peterson, Oliver, Whiteside, Operskalski, \& Ludman, 2013). The researchers found that even after variables were taken into account such as age, sex, treatment history, and overall depression severity, responses to the question addressing suicidal ideation was a strong predictor of suicide attempt. The thirteen percent of patients who marked high levels of suicidal ideation (more than half the days in a week), accounted for fifty-three percent of suicide attempts and fifty-four percent of suicide deaths among the over 84,000 responders. They found that among those reporting high rates of suicidal ideation, the risk increased over several days and persisted for several months. This indicates that suicidal ideation is an enduring vulnerability rather than a short-term crisis.

Another study specifically looking at youth found that suicidal ideation was associated with adolescent suicide (Brent, Perper, Moritz, Allman, Friend, Roth, Schweers, Balach, \& Baugher, 1993). The researchers conclusion was that effective treatment options need to be developed for youth with risk profiles. This included major depression, substance abuse, previous suicide attempts and suicidal ideation among others. Hershberger, Pilkington, and Augelli (1997) also found that suicidal ideation was among the strongest predictors of suicide attempts in their study of 194 lesbian, gay, and bisexual youth, ages 15 through 21 . Knowing that suicidal ideation is tied closely to suicidal behaviors provides helping professionals and 
practitioners with additional knowledge that can better inform preventative programming and supports for youth and adults.

Given the prevalence and incidence of suicide and suicidal ideation among LGBTQ youth (Malley, Posner, \& Potter, 2008), there is a need to explore ways to both prevent and understand suicidal behavior for this specific population. The use of mindfulness practices has been demonstrated in recent research to have a protective relationship against suicidal ideation and teaching mindfulness techniques can be an important tool to prevent suicide in at-risk populations, like LGBTQ youth (Pistorello, Fruzzetti, MacLane, Gallop, \& Iverson, 2012).

\section{Mindfulness Practice}

Mindfulness practice can be described as paying attention to what is happening right now-in the present moment (Brown and Ryan, 2003). The intention of mindfulness practice is to teach people to pay attention to what they are experiencing, moment by moment, without judgment (Kabat-Zinn, 1994). The individual engaged in mindfulness practice may then begin to notice when the mind has wandered and decide, without judgment if that is where they want it to be or go (Mark, Williams, \& Swales, 2004). Three axioms of mindfulness have been proposed by Shapiro, Carlson, Astin, and Freedman (2006), including: “on purpose" (intention), "paying attention" (attention), and "in a particular way" (attitude). These are the interwoven components of mindfulness that simultaneously make up the moment-to-moment process an individual is experiencing.

Mindfulness practice allows for one to view his or her moment-by-moment experience with increased clarity and objectivity. Mindfulness creates a shift in perspective where instead of 
being immersed in a personal narrative, we instead stand back and simply witness it. The practice of mindfulness allows for continued developing capacity for objectivity concerning one's own internal experience (Shapiro, Carlson, Astin, \& Freedman, 2006).

In the past two decades research on mindfulness has significantly increased, specifically in the area of psychological health. One of the benefits of incorporating mindfulness practice is that it may reduce depression and anxiety (Le \& Gobert, 2013). Mindfulness practice has also been associated with a decrease in ruminative thinking, which will lead to a decrease in suicidal ideation (Deyo, Wilson, Ong, \& Koopman, 2009). Ruminative thinking is the process of continuously going over a problem or thought, specifically surrounding an upsetting situation. Being in the present moment disrupts worry and rumination, potential benefits of mindfulness practices (Jain, Shapiro, Swanik, Roesch, Mills, Bell, \& Schwartz, 2007). Gayner and colleagues (2012) have even shown that mindfulness practice can improve mental health and quality of life.

\section{Mindfulness Practice and Suicidal Ideation}

Knowing the impact suicidal behaviors have on youth populations, specifically LGBTQ youth, and the suggested positive benefits mindfulness practices can have, it could be beneficial to determine how mindfulness practice may impact suicidal ideation in LGBTQ youth. There is a lack of research that looks at mindfulness practice as an intervention tool with LGBTQ youth. Although, an important study regarding mindfulness as a prevention/intervention for youth suicide that was done by Le and Gobert (2013), looked at whether a mindfulness-based program could be used as a suicide prevention tool with Native American youth. It was a small pilot study done with youth ages 15-20 that suggested further research would be useful. The researchers 
found participants experienced improved self-regulation, less mind wandering, and decreased suicidal thoughts. The overall conclusion was that mindfulness-based intervention could be suitable and adapted to fit the culture of Native American youth (Le and Gobert, 2013). This is valuable and powerful information, because it indicates mindfulness practice is culturally relevant and community created. Native American youth (ages 15-24) have been estimated to have the highest suicide rate of any cultural or ethnic group in the United States. It is important that suicide prevention programs ensure the use of culturally relevant and adaptable tools, such as mindfulness practice.

The studies that are looking at mindfulness have primarily focused on lowering relapse rates in depression. There have been over 125 clinical studies done prior to 2013 that have shown mindfulness to be effective in trials with adults with conditions such as depression and anxiety (Le \& Gobert, 2013). There have been fewer studies done specifically on mindfulness and reducing suicidal ideation and other related factors such as ruminative thinking. These studies by Mark Williams and his associates (2006) used MBCT - mindfulness based cognitive therapy. This method was developed by Zindel Segal and Mark Williams, and was partially based on the mindfulness-based stress reduction (MBSR) program created by Jon Kabat-Zinn (1994). The program incorporates eight weekly classes, with daily homework and mindfulness practice. The focus of the therapy is the practice of mindfulness as an approach to whatever occurs in a person's daily life (Mark, Williams, \& Swales, 2004), so it is extremely adaptable to fit the needs of the individual participating in the practice.

Forkmann and coauthors (2014) also used this approach and analyzed whether the effect of MBCT on suicidal ideation was dependent on other factors such as a reduction of 
worry/rumination, reduction of depressive symptoms, or an increase in mindfulness. Change in depression, change in worry, change in rumination, and change in mindfulness were all tracked in their study. It followed the MBCT format of a group-based eight week meditation exercise. Sessions included guided meditation, experiential exercises, and discussion. In addition to the weekly group sessions, participants received CDs with guided exercises and were assigned daily homework exercises ( 30 to 60 minutes daily). The study found that changes in suicidal ideation were independent from changes in depression. It also found that the impact of MBCT on suicidal ideation may have been mediated by the reduction of worry. This is incredibly important information in formatting prevention programming for youth. Forkmann and associates (2014) have found that it is not a reduction in depression levels that necessarily decreases suicidal ideation, but simply a coping skill for it. By reducing worry and the cycling of ruminative thinking, mindfulness practice is a tool to stop the suicidal crises that suicidal ideation can bring. Mindfulness practice can be an applicable tool because it allows individuals to manage self-destructive thoughts and emotions, and increases tolerance to sit with difficult thoughts and emotions. Individuals are able to perceive and experience thoughts and feelings objectively (Le \& Gobert, 2013). Other works suggest that further evidence is necessary, but pilot work indicates that $\mathrm{MBCT}$ is a promising intervention for individuals who have experienced suicidal ideation (Williams, Dugan, Crane, \& Fennell, 2006). These mindfulness meditation techniques enhanced participants ability to practice moment-by-moment awareness, and use other mindfulness techniques to interrupt the cognitive processes that fuel suicidal crises.

\section{Future Directions}


We know LGBTQ youth are at increased risk of dying by suicide. We also know there is a need to learn more about the contributing factors and the factors that may interrupt suicidal behaviors such as suicidal ideation and mindfulness. I suggest a study be performed looking at mindfulness practice as a way to decrease suicidal ideation in LGBTQ youth. The aim of this study would be to determine how mindfulness may impact suicidal ideation and related factors, with the hope of learning more about a potential tool to reduce factors associated with suicidal behaviors. This study's overall objective would be to determine the impact of mindfulness practice on suicidal ideation in LGBTQ youth.

This is a tool that can then be implemented into suicide prevention programs. This has implications for school settings, parenting education programs, as well as mental health settings and organizations serving LGBTQ youth. Mindfulness practice can be used as a protective factor for these youth, and can be implemented into suicide prevention programs in many settings. The Suicide Prevention Resource Center (2008) found that current programs serving LGBTQ youth include issues such as school safety, health promotion, violence and harassment prevention, civil rights, peer education, emergency support, connecting young people with supportive adults, and HIV and AIDS prevention and support. They do not explicitly address suicide prevention, but these services do strengthen protective factors, whether or not suicide prevention is the stated goal. Mindfulness practice can be implemented into these programs as another protective factor and another coping skill for these youth and their families.

Specific suicide prevention programs - most often implemented in schools, communities, and health care systems - can improve their ability to serve the specific needs of LGBTQ youth (Suicide Prevention Resource Center, 2008). Steps may include providing information about 
LGBTQ youths' risk for suicidal behaviors, identifying LGBTQ-inclusive services and providers, emphasizing protective factors relevent to LGBTQ youth, and supporting parents and family members of LGBTQ youth.

\section{Conclusion}

Given the prevalence and devastating impact of suicide and suicidal ideation among LGBTQ youth, it is imperative that suicide prevention expands its framework of strategies. Effective ways to prevent suicide attempts and deaths is essential. I have brought together research on suicidal ideation and mindfulness practice, specificilly in application to LGBTQ youth. A future study could lead to more support and further research in the area of mindfulness practice with LGBTQ youth for the prevention of suicide. Implementing mindulness practice into programs for LGBTQ youth would provide an additional protective factor and coping skill to potentially reduce suicidal ideation and related factors. Current suicide prevention programs can also be revised to include information relevent to LGBTQ youth. 


\section{References}

American Association of Suicidology. (2016). Suicidal behavior among lesbian, gay, and bisexual youth, 2016 youth. Retrieved from: http://www.suicidology.org/resources/lgbt

Brent, D. A., Perper, J. A., Moritz, G., Allman, C., Friend, A. M. Y., Roth, C., ... \& Baugher, M. (1993). Psychiatric risk factors for adolescent suicide: a case-control study. Journal of the American Academy of Child \& Adolescent Psychiatry, 32(3), 521-529.

Brown, K.W., Ryan, R.M. (2003). The benefits of being present: mindfulness and its role in psychological well-being. Journal of Personality and Social Psychology, 84(4), 822-848. doi:10.1037/0022-3514.84.4.822

Cash, S. J., \& Bridge, J. A. (2009). Epidemiology of youth suicide and suicidal behavior. Current opinion in pediatrics, 21(5), 613.

Center for Disease Control and Prevention (CDC). (2016). Youth risk behavior surveillanceunited states, 2015. Retrieved from: https://www.cdc.gov/healthyyouth/data/yrbs/index.htm

Deyo, M., Wilson, K.A., Ong, J., Koopman, C. (2009). Mindfulness and rumination: does mindfulness training lead to reductions in the ruminative thinking associated with depression? Explore, 5(5), 265-271. doi: 10.1016/j.explore.2009.06.005

Forkmann, T., Wichers, M., Geschwind, N., Peeters, F., van Os, J., Mainz, V., \& Collip, D. (2014). Effects of mindfulness-based cognitive therapy on self-reported suicidal ideation: results from a randomised controlled trial in patients with residual depressive symptoms. Comprehensive psychiatry, 55(8), 1883-1890.

This study investigated the effects of mindfulness based cognitive therapy (MBCT) on suicidal ideation in 130 participants with residual depressive symptoms in a randomised controlled trial. The study analyzed whether the effect of MBCT on suicidal ideation was 
dependent on other factors such as a reduction of worry/rumination, reduction of depressive symptoms, or an increase in mindfulness. Change in depression, change in worry, change in rumination and change in mindfulness were all tracked. The study found that changes in suicidal ideation were independent from changes in depression. It also found that the impact of MBCT on suicidal ideation may have been mediated by reduction of worry.

Gayner, B., Esplen, M.J., DeRoche, P., Wong, J., Bishop, S., Kavanagh, L., Butler, K. (2012). A randomized controlled study of mindfulness-based stress reduction to manage affective symptoms and improve quality of life of gay men living with HIV. Journal of Behavioral Medicine, 35(3), 272-285.

Hershberger, S. L., Pilkington, N. W., \& D'Augelli, A. R. (1997). Predictors of suicide attempts among gay, lesbian, and bisexual youth. Journal of Adolescent Research, 12(4), 477-497.

Jain, S., Shapiro, S.L., Swanick, S., Roesch, S.C., Mills, P.J., Bell, I., Schwartz, E.R. (2007). A randomized controlled study of mindfulness meditation versus relaxation training: effects on distress, positive states of mind, rumination, and distraction. Annals of Behavioral Medicine, 33, 11-21. Retrieved from: https://doi.org.proxy.lib.pdx.edu/10.1207/S15324796abm3301_2

Kabat-Zinn, J. (1994). Wherever you go, there you are: Mindfulness meditation in everyday life. NewYork: Hyperion

Le, T.N., Gobert, J.M. (2013). Translating and implementing a mindfulness-based youth suicide prevention intervention in a Native American community. Journal of Child and Family Studies, 24, 12-23. doi: 10.1007/s10826-013-9809-z 
Little, T.D., Roche, K.M., Chow, S.M., Schenck, A.P., Byam, L.A. (2016). National institutes of health pathways to prevention workshop: advancing research to prevent youth suicide. Annals of Internal Medicine, 165, 795-799. doi: 10.7326/M16-1568

Liu, R. T., \& Mustanski, B. (2012). Suicidal ideation and self-harm in lesbian, gay, bisexual, and transgender youth. American journal of preventive medicine, 42(3), 221-228.

Luoma, J.B., Villatte, J.L. (2011). Mindfulness in the treatment of suicidal individuals. Cognitive and Behavioral Practice, 19, 265-276.

Malley, E., Posner, M., \& Potter, L. Suicide Prevention Resource Center. (2008). Suicide risk and prevention for lesbian, gay, bisexual, and transgender youth. Newton, MA: Education Development Center, Inc.

Mark, J., Williams, G., Swales, M. (2004). The use of mindfulness-based approaches for suicidal patients. Archives of Suicide Research, 8(4), 315-329. doi: 10.1080/13811110490476671

This article describes and examines and similarities and differences in the use of mindfulness-based approaches in the treatment of individuals at risk for suicidal behavior who are being treated for borderline personality disorder or to reduce relapse in recurrent major depression. It describes in detail the methods of mindfulness-based cognitive therapy (MBCT). MBCT has shown to reduce relapse in depression, and the link between suicidal behavior and depression suggests MBCT could be useful for suicidal patients.

Oregon Health Authority. (2017). Youth Suicide Intervention and Prevention Plan Annual Report. Retrieved from: https://apps.state.or.us/Forms/Served/le8874c.pdf

Oregon Health Authority. (2012). Suicide, Suicide Attempts, and Ideation among Adolescents in Oregon. Retrieved from: https://www.oregon.gov/oha/PH/DiseasesConditions/InjuryFatalityData/Documents/Suici 
de,suicide\%20Attempts,\%20and\%20ideation\%20among\%20Adolescents\%20in\%20Oreg on\%202010.pdf

Pistorello, J., Fruzzetti, A.E., MacLane, C., Gallop, R., Iverson, K.M. (2012). Dialectical behavior therapy (DBT) applied to college students: a randomized clinical trial. Journal of Consulting and Clinical Psychology, 80(6), 982-994. doi: http://dx.doi.org.proxy.lib.pdx.edu/10.1037/a0029096

Safren, S. A., \& Heimberg, R. G. (1999). Depression, hopelessness, suicidality, and related factors in sexual minority and heterosexual adolescents. Journal of Consulting and Clinical Psychology, 67(6), 859-866.

Shapiro, S. L., Carlson, L. E., Astin, J. A., \& Freedman, B. (2006). Mechanisms of mindfulness. Journal of clinical psychology, 62(3), 373-386.

Simon, G.E., Rutter, C.M., Peterson, D., Oliver, M., Whiteside, U., Operskalski, B, Ludman, E.J. (2013). Does response on the PHQ-9 depression questionnaire predict subsequent suicide attempt or suicide death? Psychiatric Services, 64(12), 1195-1202. Retrieved from: http://ps.psychiatryonline.org.proxy.lib.pdx.edu/doi/pdf/10.1176/appi.ps.201200587

Suicide Prevention Resource Center. (2008). Suicide risk and prevention for lesbian, gay, bisexual, and transgender youth. Newton, MA: Education Development Center, Inc.

Williams, J. M. G., Duggan, D. S., Crane, C., \& Fennell, M. J. (2006). Mindfulness-Based cognitive therapy for prevention of recurrence of suicidal behavior. Journal of clinical psychology, 62(2), 201-210. 